\title{
Photochromism-Fret (phFRET): Modulation of Fluorescence Resonance Energy Transfer by A Photochromic Acceptor
}

\author{
Elizabeth A. Jares-Eruman , Loling Song \& Thomas M. Jovin
}

To cite this article: Elizabeth A. Jares-Eruman, Loling Song \& Thomas M. Jovin (1997) Photochromism-Fret (phFRET): Modulation of Fluorescence Resonance Energy Transfer by A Photochromic Acceptor, Molecular Crystals and Liquid Crystals Science and Technology. Section A. Molecular Crystals and Liquid Crystals, 298:1, 151-159, DOI: $\underline{10.1080 / 10587259708036155}$

To link to this article: http://dx.doi.org/10.1080/10587259708036155

Published online: 24 Sep 2006.

Submit your article to this journal ¿

Џ Article views: 34

Q View related articles ¿

Citing articles: 3 View citing articles 5 
Mol. Crist. Liq. Cryst., 1997, Vol. 298, pp. 151-159

Reprints available directly from the publisher

Photocopying permitted by license only
(C) 1997 OPA (Overseas Publishers Association)

Amsterdam B.V. Published in The Netherlands under license by Gordon and Breach Science Publishers

\title{
PHOTOCHROMISM-FRET (phFRET): MODULATION OF FLUORESCENCE RESONANCE ENERGY TRANSFER BY A PHOTOCHROMIC ACCEPTOR
}

\author{
ELIZABETH A. JARES-ERIJMAN, LOLING SONG and THOMAS M. JOVIN \\ Department of Molecular Biology, Max Planck Institute for Biophysical Chemistry, \\ P.O. Box 2841, D-37018 Göttingen, Germany
}

\begin{abstract}
We have designed a new method for the quantitative determination of fluorescence resonance energy transfer (FRET) based on the modulation of the fluorescence emission of a donor by a photochromic acceptor. Light induces a change in the structure and thus the absorption properties of the acceptor. Only one of the absorption spectra overlaps with the emission of the donor, thereby providing the means for switching the process of FRET on and off reversibly. A direct evaluation of the change in the donor quantum yield due to FRET is possible. A test molecule was devised consisting of a Lucifer Yellow donor bound covalently to a 6-nitroBIPS acceptor via a cysteine linker molecule. Photoisomerisation of the acceptor by nearUV irradiation from the spiropyran to the merocyanine form potentiated the FRET process, as evidenced by quenching of the donor. This effect disappeared upon thermal reversion to the initial spiropyran form. Photokinetic simulations were also carried out.
\end{abstract}

\section{INTRODUCTION}

Fluorescence resonance energy transfer (FRET) is a physical process by which energy is transferred nonradiatively from an excited molecular fluorophore (donor) to another chromophore (acceptor) via intermolecular long-range dipole-dipole coupling ' ${ }^{1}$ The FRET efficiency varies with the 6th power of the donor-acceptor separation over the range of $\sim 15-100 \AA$; the critical distance for $50 \%$ transfer $\left(R_{o}\right)$ requires favorable spectral overlap between the emission of the donor and the absorption of the acceptor. FRET is frequently used in biology as a spectroscopic ruler in order to detect interactions at the molecular level, and its potential in cell biology has been reviewed recently ${ }^{2,3}$. In the microscope, FRET provides information about molecular proximity in the $\mathrm{nm}$ range characteristic of the specific binding and structural interactions mediating most cellular activities, i.e. far exceeding the optical resolution $(-0.3 \mu \mathrm{m})$ of most instruments. That is, the evaluation of molecular association within a given pixel or voxel is based on the underlying photophysical process (FRET) and not the constraints imposed by the optical system per se.

There are two fundamental problems associated with quantitative FRET determinations in the microscope: (i) the local donor and acceptor concentrations and 
consequently their ratio are generally unknown; thus, the formalism for estimating the FRET efficiency $E$ must be appropriate for arbitrary stoichiometries; and (ii) for some preparations, e.g. living cells, continuous methods of observation are required. Condition (i) can be satisfied by various combinations of the donor and sensitized emission signals but at least three separate images are required ${ }^{4}$. We have developed and applied alternative techniques for FRET microscopy based on fluorescence lifetime imaging (FLIM) and photobleaching protocols (pbFRET) ${ }^{4-9}$. Condition (i) is met by evaluating $E$ via the quenching of the donor, assessed from the relative fluorescence lifetime (FLIM), relative photobleaching kinetics (pbFRET), or normalized donor intensity. In all three cases, a reference state for the unquenched donor is required, i.e. one in which the acceptor is absent. This can be achieved in the case of pbFRET by preparing a corresponding separate but comparable sample or from a (different) region in which the acceptor is removed photodestructively ${ }^{7}$. The latter approach also serves for FRET estimations within a given region in the case of fluorescence lifetime or relative donor emission measurements ${ }^{7}$.

In this communication, we describe a new procedure for FRET microscopy in which the acceptor is created and removed locally in a reversible manner, thus potentially satisfying the above condition (ii) as well as (i). The two probes are selected according to their spectroscopic properties, such that only one of the photochromic forms of the acceptor is competent for the transfer of excitation energy from the donor. The fluorescence emission of the donor is modulated by systematic photochemical manipulation of a photochromic acceptor. That is, the donor fluorescence is measured in the "presence" and "absence" of the acceptor, the latter case corresponding to the reference state described above.

\section{MATERIALS AND METHODS}

\section{Chemicals and Methods}

The spirobenzopyran (1-carboxyethyl-3,3-dimethyl-6-nitrospiro-[indoline-2,2'[2H]benzopyran]) (6-nitroBIPS) was obtained from Chroma and purified by recrystalization in ethanol. $\mathrm{N}$-(iodoacetamido-2- $\mathrm{N}$-ethyl)-4-amino-3,6-disulfo-1,8naphtalimide (Lucifer Yellow iodoacetamide) was from Molecular Probes. Cysteine and N,N-bis[2-hydroxyethyl]-glycine (bicine) were from Sigma.

The model molecule LY-BIPS (Figure 1) was prepared by reaction of cysteine with Lucifer Yellow iodoacetamide at room temperature in 0.1 M TEAA (triethylammonium acetate, $\mathrm{pH} \mathrm{7)}$ for $2 \mathrm{~h}$. The solution was then equilibrated at $\mathrm{pH} 9(1 \mathrm{M}$ bicine buffer $)$ and reacted at room temperature for $1 \mathrm{~h}$ by subsequent addition of 6-nitroBIPS succinimidyl 
ester (in DMF). The product was purified by HPLC (reversed phase RP-18, 35/65 $\mathrm{CH}_{3} \mathrm{CN} / 1 \mathrm{M}$ TEAA).

\section{Spectroscopy}

Absorption spectra were acquired with a Kontron Uvicon 820 spectrophotometer at room temperature and with $1 \mathrm{~nm}$ resolution. Steady-state fluorescence measurements were performed with an SLM 8000S spectrofluorimeter at $4^{\circ} \mathrm{C}$ and with $1 \mathrm{~nm}$ resolution. The emission spectra were corrected for instrument response, lamp fluctuations and solvent background contributions. Polarization artifacts were avoided by using "magic angle" conditions. Emission spectra were collected in the $440-700 \mathrm{~nm}$ range with excitation at $430 \mathrm{~nm}$ (donor), using constant slit apertures and gain settings.

\section{FRET Formalism}

The FRET efficiency $E$ varies as a function of the donor-acceptor separation $\left(r_{d a}\right)$ normalized by the characteristic transfer distance $\left(R_{o}\right)$ for $50 \%$ transfer ${ }^{1,10}$.

$$
E=\left[1+\left(\frac{r_{d u}}{R_{o}}\right)^{6}\right]^{-1} \text { where } \quad R_{o}^{6}=8.79 \cdot 10^{-28} \cdot J n^{-4} \kappa^{2} \Phi_{d}
$$

$J$ is the spectral overlap integral, $n$ the refractive index of the medium separating donor and acceptor, $\boldsymbol{\kappa}$ the donor-acceptor orientation factor, and $\Phi_{d}$ the donor emission quantum yield in the absence of FRET.

The determination of $E$ is based on the evaluation of donor quenching from a comparison of signals in the donor emission region corresponding to solutions (or volume elements resolved in the microscope) of molecules containing either both donor and acceptor $\left({ }_{d a} f_{d}^{d}\right)$ or donor alone $\left({ }_{d} f_{d}^{d}\right)$.

$$
E=1-\frac{{ }_{d a} f_{d}^{d}}{{ }_{d} f_{d}^{d}}
$$

\section{RESULTS}

\section{Lucifer Yellow-BIPS, a Model System for Photochromism-FRET}

In order to test the feasibility of the method we synthesized a molecule containing a photochromic acceptor moiety covalently attached to a fluorescent donor (Figure 1). An estimation of the range of distances between the center of the donor (Lucifer Yellow) and the merocyanine acceptor was performed by energy minimization with Sybyl (AMBER 4.1 package), obtaining a range for $r_{d a}$ of $15-20 \AA$. 


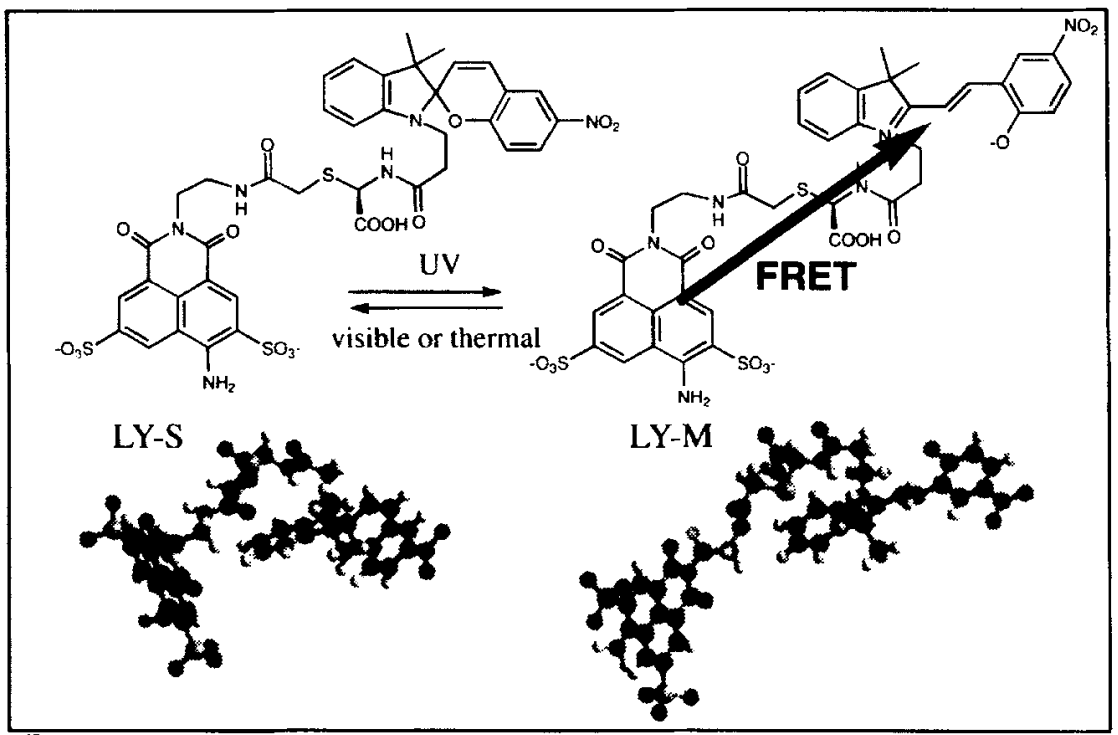

FIGURE 1 LY-BIPS model compound. See Materials and Methods.

Photochemical Conversion of the Spiropyran to the Merocyanine Form

Merocyanine formation was studied as a function of time in order to determine the condition of maximum conversion avoiding photodegradation. A final concentration of 5 : $\mu \mathrm{M}$ 6-nitroBIPS in ethanol was exposed to the UV (254 nm) at room temperature up to $20 \mathrm{~min}$. In Figure 2, the absorption spectra taken at 2, 5, 10 and $15 \mathrm{~min}$ of UV exposure are shown. The conversion to the merocyanine form reached a maximum after 15 min of UV irradiation.

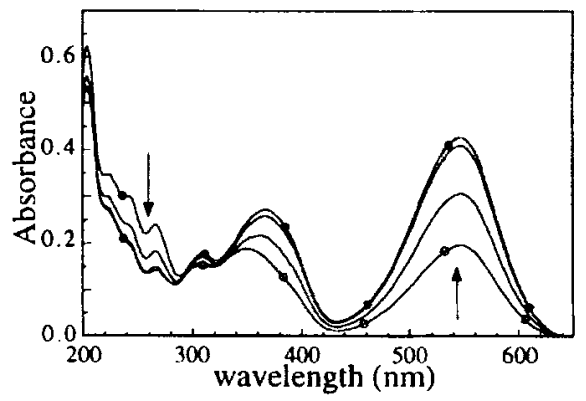

FIGURE 2 The photochemical conversion from the spiropyran to the merocyanine form as a function of the duration of UV irradiation. Absorption spectrum after a 2 $\min (O)$ and $15 \min (\bigcirc)$ exposure to UV $(254 \mathrm{~nm})$ are depicted. The arrows indicate the decrease in the spiropyran form or the increase in the merocyanine form. The initial spiropyran state is not shown (see Figure 4A). 


\section{Thermal Back Reaction}

The same solution was irradiated for $30 \mathrm{sec}$ at $254 \mathrm{~nm}$, and was allowed to recover to the spiropyran form at room temperature. The thermal reversion was monitored as a function of time. The absorbance change at the peak of the merocyanine form $(546 \mathrm{~nm})$ was fit to a monoexponential function $\left(a+b \cdot \exp \left[-k_{T}^{*} t\right]\right)$ where $a$ was the absorbance at $546 \mathrm{~nm}$ before photoconversion and/or after the thermal back reaction. The data fit to the monoexponential function very well, in agreement with other findings ${ }^{11,12}$, and yielded a rate for the thermal reversion of $0.039 \mathrm{~min}^{-1}$.

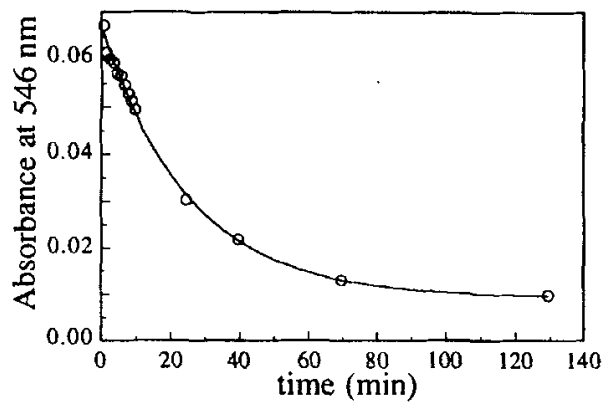

FIGURE 3 Thermal back reaction of 6-nitroBIPS merocyanine to spiropyran at room temperature. The fit to the exponential function yielded $a=0.009, b=0.058$, and $k_{T}=0.039 \mathrm{~min}^{-1}$.

\section{FRET Modulation by Changes in the Overlap Integral}

The feasibility of the proposed FRET method was tested by evaluating the change in the overlap integral calculated from the emission spectrum of the donor and the absorption spectra of the acceptor in the two photochromic forms.

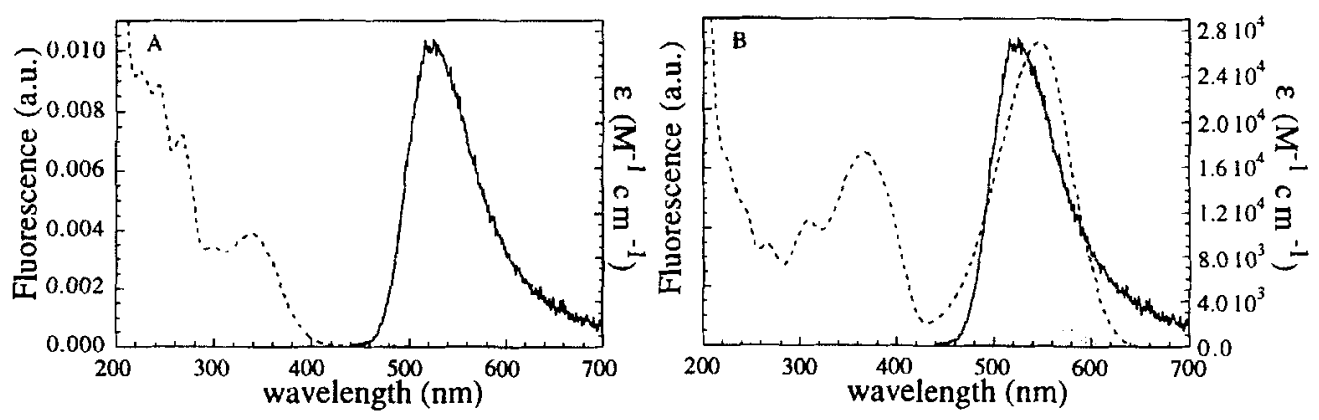

FIGURE 4 The absorption spectra (---) of 6-nitroBIPS (acceptor) in the spiropyran (A) or merocyanine form (B) compared to the emission spectrum of the Lucifer Yellow donor $(-$ ). The kernel of the overlap integral (shaded area) 10 increases substantially in $\mathbf{B}$. 
Figure 4 compares the absorption spectra of 6-nitroBIPS as the spiropyran and merocyanine forms with the fluorescence emission spectrum of Lucifer Yellow. The spiropyran exhibited negligible overlap. In contrast, the large increase in the absorption of the merocyanine form at $450-650 \mathrm{~nm}$ led to a very substantial overlap (shaded area in Figure $4 \mathrm{~B}$ ).

The FRET efficiency $E$ was then determined in the LY-BIPS molecule by evaluation of the donor quenching. Figure 5 shows the fluorescence of LY-BIPS in the spiropyran form and after conversion to the merocyanine by irradiation at $254 \mathrm{~nm}$ for $15 \mathrm{~min}$. The emission at $<600 \mathrm{~nm}$, excited at $430 \mathrm{~nm}$, originated solely from the Lucifer Yellow donor. The substantial quenching observed with the merocyanine acceptor corresponded to a transfer efficiency of 0.32 . The sample was allowed to recover spontaneously to the original spiropyran form at room temperature for $1 \mathrm{~h}$, after which the fluorescence emission regained its initial value.

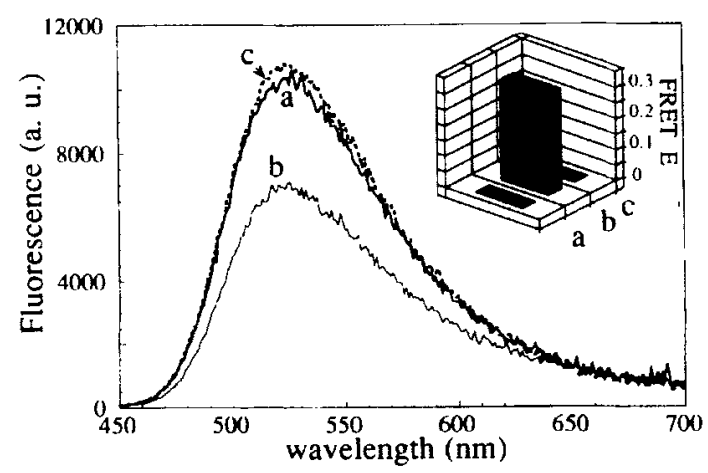

FIGURE 5 Lucifer Yellow fluorescence of LY-BIPS before (a) and after (b) lightinduced conversion of spiropyran to merocyanine. After $1 \mathrm{~h}$ at room temperature, the molecule reverted to the spiropyran form, as evidenced by the restoration of the fluorescence to its original value (c). Inset: corresponding FRET efficiency values.

\section{Photokinetic Modeling}

The combination of photochromic conversion and fluorescence resonance energy transfer gives rise to a photochemically bistable system. When the spiropyran is photoconverted to the merocyanine form, the change in absorption spectral region and consequent increase in overlap integral potentiates FRET ("on" state). On the other hand, as the merocyanine form is thermally (or photochemically) reverted back to the spiropyran form, the overlap integral decreases dramatically and thus the FRET is in the "off" state. This bistability critically depends on the modulation of the excitation irradiance of the photochromic 
compound with concomitant excitation of the fluorescent donor. The kinetic simulation presented here was designed to theoretically explore the conditions for controlling bistability and thereby providing guidance for the practical implementation of quantitative photochromism-FRET measurements in the light microscope. The photokinetic simulation demonstrates the temporal course of the major molecular forms determining the overall "on" and "off" states of the FRET system.

The generalized reaction mechanisms are depicted in Figure 6. The model takes into account only the main photokinetic events, omitting transient isomeric states and photodegradation of either the photochromic acceptor or the fluorescent donor molecules.

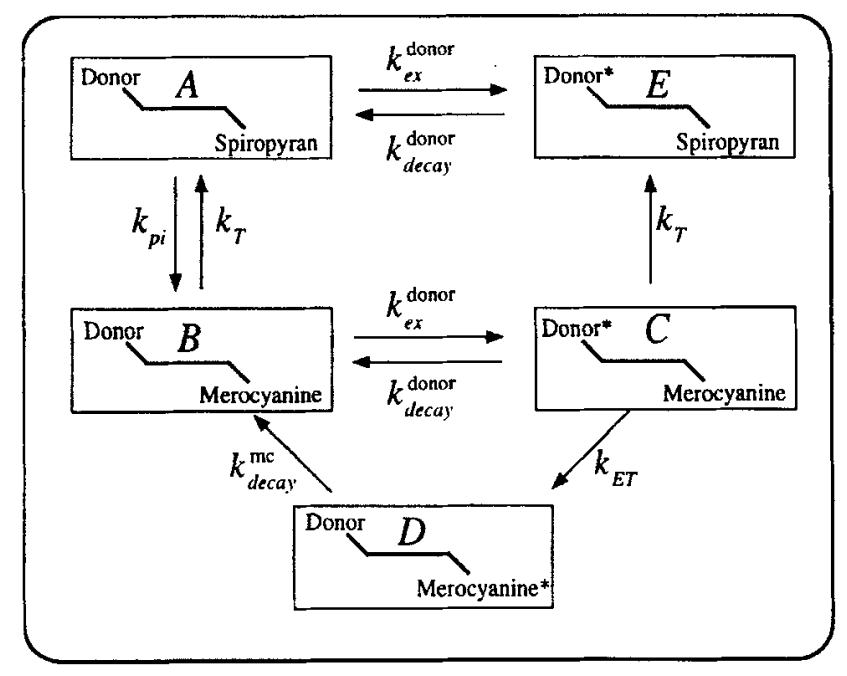

FIGURE 6 Schematic diagram of the main photokinetic events and mathematical formulation of photochromism-FRET. The excited (fluorescent) states of the donor are species $C$ and $E$, and of the merocyanine acceptor species $D$. Definitions: $k_{p i} \propto \sigma_{\mathrm{sp}}$. irradiance $(\mathrm{UV})$, rate constant for photoisomerization with an absorption cross-section of the spiropyran $=\sigma_{\mathrm{sp}} ; k_{\text {decay }}^{\mathrm{mc}}$, inverse of the singlet excited state lifetime of the merocyanine; $k_{T}$, rate constant for thermal reversion from the merocyanine to the spiropyran form; $k_{e x}^{\text {donor }}=\sigma_{\text {donor }}$. irradiance (visible), rate constant for excitation of the donor; $k_{\text {decay }}^{\text {donor }}$, inverse of the singlet excited state lifetime of the donor; $k_{E r}=k_{\text {decay }}^{\text {donor }} \cdot[E /(1-E)]$, rate constant for resonance energy transfer.

Based on this reaction scheme, a system of differential equations was formulated (Equation 3) and solved by numerical integration. The photochemical parameters and the rate constants used in the simulations were either measured, derived from our own experimental data, or extracted from the existing literature ${ }^{11.12}$ on the same photochromic 


$$
\begin{aligned}
& \frac{\mathrm{d}[A]}{\mathrm{dt}}=-k_{p i}[A]-k_{e x}^{\text {donor }}[A]+k_{T}[B]+k_{\text {decay }}^{\text {donor }}[E] \\
& \frac{\mathrm{d}[B]}{\mathrm{dt}}=-k_{T}[B]-k_{e x}^{\text {donor }}[B]+k_{p i}[A]+k_{\text {decay }}^{\mathrm{mc}}[D]+k_{\text {decay }}^{\text {donor }}[C] \\
& \frac{\mathrm{d}[C]}{\mathrm{d} \mathrm{t}}=-k_{T}[C]-k_{E T}[C]+k_{e x}^{\text {donor }}[B]-k_{\text {decay }}^{\text {donor }}[C] \\
& \frac{\mathrm{d}[D]}{\mathrm{dt}}=-k_{\text {decar }}^{\text {donor }}[D]+k_{E T}[C] \\
& \frac{\mathrm{d}[E]}{\mathrm{dt}}=-k_{\text {decay }}^{\text {donor }}[E]+k_{T}[C]+k_{e x}^{\text {donor }}[A]
\end{aligned}
$$

compound. Some of the parameters determined experimentally were also used in the photokinetic simulation depicted below (Figure 7). The visible light source required for excitation of the fluorescent donor was first turned on for $2 \mathrm{~min}$. In the absence of the merocyanine acceptor, the donor fluorescence was at a maximal level. In the second step, the UV light source was turned on for 20 min to photoconvert the acceptor from the spiropyran to the merocyanine form. The UV source was then removed and the visible excitation of the donor reactivated. The photoconverted merocyanine now functioned as an acceptor for the singlet excited state energy of the fluorescent donor, thereby leading to a quenching of the latter. The donor fluorescence recovered in a slow process (Figure 7), reflecting the thermal reversion of the merocyanine to the spiropyran form.

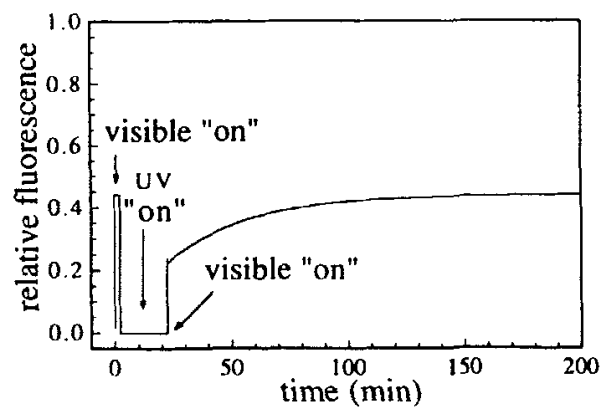

FIGURE 7 Simulation of the donor fluorescence in the absence and presence of the merocyanine form. The relative fluorescence intensity corresponds to the fraction of the total donor population in the excited state. Before UV exposure no FRET process takes place. Following UV irradiation a decrease (quenching) of the fluorescence emission of the donor due to FRET is observed. Subsequently the donor fluorescence emission recovers to the original level as the merocyanine reverses thermally to the spiropyran. 


\section{DISCUSSION}

The process of energy transfer can be activated and deactivated by inducing the conversion between spiropyran and merocyanine forms, as demonstrated by the experiments in Figures 4 and 5 . The model molecule yielded a fluorescence energy transfer efficiency of $32 \%$. For the calculated donor-acceptor separation of 15-20 $\AA$, the $R_{o}$ calculated from Equation 1 would be $13-22 \AA$. This value is approximately half of that derived from the measured parameters defining $R_{o}$ (Equation 1), using the usual assumption of $\kappa^{2}=2 / 3$. Conceivably, the orientation between the donor-acceptor moieties of the model compound LY-BIPS may not be random. In any event, this preliminary study establishes the feasibility of using bistable acceptors for reversible FRET determinations.

In addition to the anticipated uses in biological studies, the concept has implications for the field of reversible optical data storage. One can conceive of a system for nondestructive readout based on appropriate combinations of fluorescent dyes, such as thermally stable diarylalkenes or fulgides. The fluorescent dye (donor) would be excited selectively in a region of minimum absorption by the photochromic acceptor molecule.

\section{ACKNOWLEDGMENTS}

We thank Dr. Robert Bertelson for helpful discussions. L. Song is the recipient of a postdoctoral fellowship from the European Commission TMR program.

\section{REFERENCES}

1. T. Förster, Naturwissenschaften, 6,166 (1946).

2. R. Y. Tsien, B. J. Bacskai and S. R. Adams, Trends Cell Biol., 3, 242 (1993).

3. R. M. Clegg, in Fluorescence Imaging Spectroscopy and Microscopy, edited by X. F. Wang and B. Herman (John Wiley \& Sons, Inc., New York, 1996), pp. 179252.

4. T. M. Jovin and D. J. Arndt-Jovin, in Cell Structure and Function by Microspectrofluometry, edited by E. Kohen, J. G. Hirschberg and J. S. Ploem (Academic Press, London, 1989), pp 99-117.

5. T. W. J. Gadella Jr., T. M. Jovin and R. M. Clegg, Biophys. Chem., 48, 221 (1993).

6. T..W. J. Gadella Jr. and T. M. Jovin, J. Cell Biol., 129, 1543 (1995).

7. P. I. H. Bastiaens, I. V. Majoul, P. J. Verveer, H.-D. Söling and T. M. Jovin, EMBO J., 15, 4246 (1996).

8. P. I. H. Bastiaens and T. M. Jovin, Proc. Natl. Acad. Sci. USA, 93, 8407 (1996).

9. P. I. H. Bastiaens and T. M. Jovin, in Cell Biology: A Laboratory Handbook, 2nd Ed., edited by J. E. Celis (Academic Press, New York, 1996), in press.

10. E. A. Jares-Erijman and T. M. Jovin, J.Mol. Biol., 257, 597 (1996).

11. R. Guglielmetti, in Photochromism: Molecules and Systems, edited by H. Dürr and H. Bouras-Laurent (Elsevier Science Publishers, BV, 1990), pp. 314-466.

12. V. Pimienta, D. Lavabre, G. Levy, A. Samat, R. Guglielmetti and J. C. Micheau, J. Phys. Chem., 100, 4485 (1996). 